Pure and Applied Mathematics Quarterly

Volume 3, Number 4

(Special Issue: In honor of

Grisha Margulis, Part 1 of 2)

1027-1036, 2007

\title{
Linear Drift and Poisson Boundary for Random Walks
}

\author{
Anders Karlsson* and François Ledrappier ${ }^{\dagger}$ \\ For Gregory Margulis on the occasion of his sixtieth birthday
}

\begin{abstract}
We consider a nondegenerate random walk on a locally compact group with finite first moment. Then, if there are no nonconstant bounded harmonic functions, all the linear drift comes from a real additive character on the group. As a corollary we obtain a generalization of Varopoulos' theorem that in the case of symmetric random walks, positive linear drift implies the existence of nonconstant bounded harmonic functions. Another consequence is the phenomenon that for some groups (including certain Grigorchuk groups) the drift vanishes for any measure of finite first moment.
\end{abstract}

\section{INTRODUCTION}

A metric space is called proper if closed bounded sets are compact. Let $G$ be a locally compact group and $d$ a left invariant proper metric on $G$ (it is assumed throughout that the topology generated by $d$ coincides with the given one). When $G$ is second countable such a metric always exists, see [HP]. Let $\nu$ be a probability measure on $G$ of finite first moment, which means that

$$
\int_{G} d(e, g) d \nu(g)<\infty .
$$

Let $Z_{n}$ denote trajectories of the corresponding random walk, that is,

$$
Z_{n}=g_{0} g_{1} \ldots g_{n-1}
$$

where $g_{i}$ are independent random variables taking values in $G$ with distribution $\nu$. The probability distribution $\nu^{* n}$, defined as the $n$-times convolution $\nu * \nu * \ldots * \nu$ of $\nu$, is the distribution of $Z_{n}$. It is natural to assume that the support of $\nu$ generates $G$ as a group, in which case we refer to $\nu$ as nondegenerate. We are

Received April 25, 2006.

*Supported by Swedish Research Council (VR) grant 2002-4771.

${ }^{\dagger}$ Supported in part by NSF grant DMS-0500630. 
interested in asymptotic objects associated with the random walk. By Kingman's subadditive ergodic theorem, the linear drift

$$
l(\nu):=\lim _{n \rightarrow \infty} \frac{1}{n} d\left(e, Z_{n}\right)=\inf _{n} \frac{1}{n} \int_{G} d(e, g) \nu^{* n}(g)
$$

exists and is constant almost surely. A bounded measurable $f: G \rightarrow \mathbb{R}$ is $\nu$ harmonic if

$$
f(g)=\int_{G} f(g h) d \nu(h)
$$

for any $g \in G$. Constant functions are obviously $\nu$-harmonic. If $f$ is a bounded harmonic function, then $f\left(Z_{n}\right)$ is a bounded martingale and therefore converges almost surely. We say that the Poisson boundary is trivial if the constant functions are the only bounded $\nu$-harmonic functions (see $[\mathrm{KV}]$ ). Our main result is the following:

Theorem 1. Let $G$ be a locally compact group with a left invariant proper metric and $\nu$ be a nondegenerate probability measure on $G$ with first moment. Then, if the Poisson boundary is trivial, there is a 1-Lipschitz homomorphism $T: G \rightarrow \mathbb{R}$ such that for almost every trajectory $Z_{n}$ of the corresponding random walk, we have:

$$
\lim _{n \rightarrow \infty} \frac{1}{n} T\left(Z_{n}\right)=\int_{G} T(g) d \nu(g)=l(\nu) .
$$

A measure $\nu$ is symmetric if $d \nu\left(g^{-1}\right)=d \nu(g)$ for every $g \in G$. A measure is centered if every homomorphism of $G$ into $\mathbb{R}$ is centered, meaning that the $\nu$-weighted mean value of the image is 0 (cf. [G]). Every symmetric measure $\nu$ is centered, since for any homomorphism $T: G \rightarrow \mathbb{R}$, the mean value, which is

$$
\int_{G} T(g) d \nu(g)=\int_{G} T\left(g^{-1}\right) d \nu(g)=-\int_{G} T(g) d \nu(g),
$$

must hence equal 0. By simple contraposition, we get:

Corollary 2. Let $G$ be a locally compact group with a left invariant proper metric and $\nu$ be a nondegenerate centered probability measure on $G$ with first moment. Then, if $l(\nu)>0$, there exist nonconstant bounded $\nu$-harmonic functions.

Corollary 2 was proved by Varopoulos ([Va]) in the case $\nu$ is symmetric and of finite support on a finitely generated group. His proof rests on estimates for $n$-step transition probabilities of symmetric Markov chains. A simpler proof of the crucial estimate was given by Carne $[\mathrm{C}]$. See also [Al] and [M] for interesting extensions. Note however that so far these estimates do not work for measures of infinite support. Measures with infinite support and finite first moment occur for example in the Furstenberg-Lyons-Sullivan discretization procedure of the Brownian motion, see [KL2]. 
Corollary 2 may also be compared with one of the main theorems in the paper $[G]$ of Guivarc'h which states that for any connected amenable Lie group and any nondegenerate, centered measure $\nu$ with finite moments of all orders, the linear drift vanishes. The proof goes via a reduction to the case of connected, simply connected, nilpotent Lie groups. Guivarc'h pointed out to us that it is in fact proved in $[\mathrm{G}]$ that in the case of a connected amenable Lie group all the drift comes from an additive character (similar to Theorem 1 above). For finitely generated amenable groups this is no longer true: consider a simple symmetric random walk on the wreath product of $\mathbb{Z}^{3}$ with $\mathbb{Z} / 2 \mathbb{Z}$. This example has nontrivial bounded harmonic functions, hence the drift is positive, but all additive characters factor through $\mathbb{Z}^{3}$ and there the random walk moves sublinearly. In this discrete case, one should also mention the result of Kaimanovich $([\mathrm{K}])$ that when the group $G$ is polycyclic and $\nu$ is centered, then the linear drift vanishes.

In the case when $G$ is a finitely generated group, entropy theory (see Section 4) yields a kind of converse to Corollary 2 .

Corollary 3. Let $G$ be a finitely generated group and $\nu$ be a nondegenerate centered probability measure on $G$ with first moment. Then $l(\nu)>0$ if, and only if, there are nonconstant bounded $\nu$-harmonic functions on $G$.

Note that a measure may be centered for the simple reason that there are no nontrivial homomorphisms into $\mathbb{R}$, in this case Corollary 2 gives:

Corollary 4. Let $G$ be a locally compact group with a left invariant proper metric and $\nu$ be a nondegenerate probability measure on $G$ with first moment. Assume that the only bounded $\nu$-harmonic functions are the constants and that $H^{1}(G, \mathbb{R})=0$. Then $l(\nu)=0$.

The point here is that $\nu$ is not necessarily symmetric. It is remarkable that for a whole general class of groups, the nonexistence of homomorphisms can have such a strong influence on the drift; this is in great contrast with the case of nonamenable groups where no matter what, any nondegenerate measure of first moment must have positive drift (see $[\mathrm{G}]$ ).

Recall that, if $v$ be the volume growth rate of a finitely generated group $G$, $v \leq \log |S|<+\infty$, then it is a fact (see [Av], [G], [Ve] and below) that if $v=0$, then the Poisson boundary is trivial. Examples of groups with subexponential growth $(v=0)$ and no nontrivial homomorphisms into the reals include the torsion groups with subexponential (but superpolynomial) growth constructed by Grigorchuk. We may formulate: 
Corollary 5. Let $G$ be a torsion group of subexponential growth (e.g. certain Grigorchuk groups) and $\nu$ any measure with first moment. Then $l(\nu)=0$.

Indeed, let $H$ be the subgroup generated by the support of $\nu$. Since every element has finite order, any homomorphism $H \rightarrow \mathbb{R}$ vanishes and using the word metric on $G$ for $H$, the statement follows from the volume criterion and Theorem 1.

See [E] for properties of measures without first moment, but with finite entropy, on such groups of subexponential growth.

\section{Preliminaries}

2.1. Horofunctions. Let $(X, d)$ be a proper metric space. Fix a basepoint $x_{0} \in$ $X$. Let

$$
\Phi: X \rightarrow C(X)
$$

be defined by $x \mapsto d(x, \cdot)-d\left(x, x_{0}\right)$ and where the topology on the space of continuous functions $C(X)$ is uniform convergence on compact sets. It can be checked that $\Phi$ is a continuous injection, and we identify $X$ with its image. Let $H=\overline{\Phi(X)}$. It is easy to verify that $H$ is a compact and metrizable space. The points in $H \backslash \Phi(X)$ are called horofunctions (based at $x_{0}$ ).

The action by $\operatorname{Isom}(X, d)$ on $X$ extends continuously to an action by homeomorphisms of $H$ and is given by

$$
g \cdot h(x)=h\left(g^{-1} x\right)-h\left(g^{-1} x_{0}\right) .
$$

Note that we always have

$$
|h(x)| \leq d\left(x, x_{0}\right) \text { and }|h(x)-h(y)| \leq d(x, y) .
$$

See [KL1] and the references therein for more information.

2.2. A noncommutative ergodic theorem. Let $(\Omega, \mu)$ be a standard Borel space with $\mu(\Omega)=1$ and $L: \Omega \rightarrow \Omega$ an ergodic measure preserving transformation. Given a measurable map $g: \Omega \rightarrow \operatorname{Isom}(X, d)$ (where the measurable structure on $\operatorname{Isom}(X, d)$ is the Borel structure coming from the topology of convergence on compact sets), let

$$
Z_{n}(\omega)=g(\omega) g(L \omega) \ldots g\left(L^{n-1} \omega\right) .
$$

Random walks $(G, \nu)$ is a special case of this setting as follows: $(\Omega, \mu)$ is the infinite product measure space of copies of $(G, \nu)$ indexed by $n \in \mathbb{Z}, L$ is the shift transformation and $g$ is simply the projection on the first coordinate $n=0$. The 
measure $\nu$ equals $g_{*} \mu$. The metric space $X$ is the group $G$ with the left invariance distance $d$, and the action is by left translations of $G$ on itself. The base point $x_{0}$ is the group identity $e$ in the special case. The linear drift is

$$
l=\lim _{n \rightarrow \infty} \frac{1}{n} d\left(Z_{n}(\omega) x_{0}, x_{0}\right)
$$

which exists by the subadditive ergodic theorem and coincides with the previous definition in the special case.

In this general ergodic setting we proved in [KL1] that

Theorem 6. There is a measurable map $h .: \Omega \rightarrow H$, almost everywhere defined, such that

$$
\lim _{n \rightarrow \infty}-\frac{1}{n} h_{\omega}\left(Z_{n}(\omega) x_{0}\right)=l
$$

One of the more general previous theorems of this type was obtained in [KM] by Margulis and the first author proving a statement equivalent to Theorem 6 in the case of (even nonproper) nonpositively curved metric spaces. See [KL1] for references to further previous results.

2.3. Poisson boundary and stationary measures. Let $\nu$ be a probability measure on a topological group $G$. Assume $G$ acts on a space $K$ with measure $\eta$. The convolution measure on $K$ is defined by

$$
\nu * \eta(A)=\int_{g \in G} \eta\left(g^{-1} A\right) d \nu(g) .
$$

The measure $\eta$ is called $\nu$-stationary if $\nu * \eta=\eta$. Assume that there are no nonconstant bounded $\nu$-harmonic functions. Then, as is well-known, $\eta$ is in fact $G$-invariant. Indeed, given a continuous function $f$ on $K$ it follows from the stationarity relation that

$$
F(g):=\int_{K} f(g z) d \eta(z)=\int_{K} f(z) d\left(g_{*} \eta\right)(z)
$$

is a bounded $\nu$-harmonic function, hence constant. Since this holds for all continuous functions $f$, we have that $\eta$ must be invariant.

\section{Proof of Theorem 1}

Define the skew-product system $\bar{L}: \Omega \times H \rightarrow \Omega \times H$ by

$$
\bar{L}(\omega, h)=\left(L \omega, g(\omega)^{-1} h\right) .
$$


The proof of Theorem 6 in [KL1] goes by constructing a $\bar{L}$-invariant measure $\eta$ on $\Omega \times H$ such that, if $\bar{F}(\omega, h):=-h\left(\left(g(\omega)^{-1} x_{0}\right)\right.$, we have:

$$
l(\nu)=\int \bar{F}(\omega, h) d \eta(\omega, h) .
$$

The measure $\eta$ is constructed as a weak limit of

$$
\frac{1}{n} \sum_{i=0}^{n-1}\left(\bar{L}^{i}\right)_{*} \mu_{n}
$$

where the disintegration of $\mu_{n}$ is given by

$$
\mu_{n, \omega}=\delta_{\Phi\left(Z_{n}(\omega)\right)} .
$$

Lemma 7. Assume that there is no nonconstant bounded harmonic function. Then, the measure $\eta$ is a product measure $\mu \times m$, where $m$ is a $G$-invariant measure on $H$.

Proof. Let $m$ denote the projection of $\eta$ on $H$, in other words $m(B)=\eta(\Omega \times B)$.

Step 1: The measure $m$ is $\nu$-stationary. Indeed, note that

$$
\begin{aligned}
\left(\bar{L}^{i}\right)_{*} \mu_{n}(\Omega \times B) & =\int_{\Omega} \int_{H} 1_{B}\left(Z_{i}^{-1}(\omega) h\right) \mu_{n, \omega}(d h) \mu(d \omega) \\
& =\int_{\Omega} \delta_{\Phi\left(Z_{n}(\omega)\right)}\left(Z_{i}(\omega) B\right) \mu(d \omega) \\
& =\int_{\Omega} \delta_{\Phi\left(x_{0}\right)}\left(Z_{n-i}^{-1}\left(L^{i} \omega\right) B\right) \mu(d \omega) \\
& =\nu^{*(n-i)} \times \delta_{\Phi\left(x_{0}\right)}(B) .
\end{aligned}
$$

In view of this it is clear that $\nu * m=m$.

Step 2: Negative coordinates in $\Omega$. Let $\Omega_{-}$be the product of copies of $(G, \nu)$ indexed by the negative integers. The natural projection of the measure $\eta$ on $\Omega_{-} \times H$ is the product measure $\mu_{-} \times m$, because the above construction of $\eta$ was done independently of the negative coordinates. Given $\omega_{-} \in \Omega_{-}$, the distribution of $h$ is just the projection of $\eta$ on $H$, which is $m$ by definition.

Step 3: Coordinates up to $k$ in $\Omega$. We compute now the natural projection of the measure $\eta$ to the product of $H$ and of copies of $(G, \nu)$ indexed by integers 
smaller than $k$, for $k>0$. Write, for bounded measurable $F_{1}, F_{2}$ :

$$
\begin{aligned}
\int F_{1}\left(\omega_{n}, n<k\right) & F_{2}(h) d \eta=\int\left(F_{1} F_{2}\right)\left(\bar{L}^{-k}(\omega, h)\right) d \eta \\
& =\int\left(F_{1} F_{2}\right)\left(L^{-k} \omega, Z_{k}\left(L^{-k} \omega\right) h\right) d \eta \\
& =\int F_{1}\left(L^{-k} \omega\right) F_{2}\left(Z_{k}\left(L^{-k} \omega\right) h\right) d \eta(\omega, h) \\
& =\int F_{1}\left(L^{-k} \omega\right)\left(\int F_{2}\left(Z_{k}\left(L^{-k} \omega\right) h\right) d m(h)\right) d \mu(\omega),
\end{aligned}
$$

where we used Step 2 to write the last line, because $F_{1}\left(L^{-k} \omega\right)$ and $Z_{k}\left(L^{-k} \omega\right)$ both depend only on negative coordinates of $\omega$. Since there are no nonconstant bounded harmonic functions, the stationary measure $m$ is invariant, and

$$
\int F_{2}\left(Z_{k}\left(L^{-k} \omega\right) h\right) d m(h)=\int F_{2} d m,
$$

and we find $\int F_{1}\left(\omega_{n}, n<k\right) F_{2}(h) d \eta=\int F_{1} d \mu \int F_{2} d m$, as claimed.

Letting $k$ go to $+\infty$ in Step 3 proves the Lemma.

By Lemma 7 and section 2.3, assuming that there are no nonconstant bounded harmonic functions, we find a $G$-invariant probability measure $m$ on $H$ satisfying:

$$
l(\nu)=-\int h\left((g(\omega))^{-1} x_{0}\right) d m(h) d \mu(\omega)=-\int h\left(g^{-1}\right) d m(h) d \nu(g) .
$$

Set

$$
T(g)=-\int h\left(g^{-1}\right) d m(h)
$$

The integral makes sense because $\left|h\left(g^{-1}\right)\right| \leq d(g, e)$. The function $T: G \rightarrow \mathbb{R}$ is measurable and satisfies $|T(g)| \leq d(g, e)$ and $l(\nu)=\int T(g) d \nu(g)$.

Recall that the action of $G$ on $H$ is given by:

$$
\gamma_{1} \cdot h\left(\gamma_{2}\right)=h\left(\gamma_{1}^{-1} \gamma_{2}\right)-h\left(\gamma_{1}^{-1}\right)
$$

so that:

$$
\begin{aligned}
T\left(g g^{\prime}\right) & =-\int h\left(g^{\prime-1} g^{-1}\right) d m(h) \\
& =-\int g^{\prime} \cdot h\left(g^{-1}\right) d m-\int h\left(g^{\prime-1}\right) d m \\
& =T(g)+T\left(g^{\prime}\right),
\end{aligned}
$$


where we used the invariance of $m$ in the last equation. Hence the map $T$ defines a homomorphism

$$
T: G \rightarrow \mathbb{R},
$$

in particular $T\left(g^{-1}\right)=-T(g), T$ is $\nu$-integrable, and (1) becomes

$$
l(\nu)=\int_{\Omega} T(g(\omega)) d \mu(\omega)=\int_{G} T(g) d \nu(g) .
$$

Finally we note that $T$ moreover is a 1-Lipschitz map:

$$
\left|T(g)-T\left(g^{\prime}\right)\right| \leq \int\left|h\left(g^{-1}\right)-h\left(g^{\prime-1}\right)\right| d m(h) \leq \int d\left(g, g^{\prime}\right) d m(h)=d\left(g, g^{\prime}\right) .
$$

\section{Entropy AND PRoOF of COROLlary 3.}

In this section $G$ is a finitely generated group. Let $S$ be a symmetric finite generating set. The distance on $G$ is the corresponding left invariant word metric $|\cdot|$. It is clearly a proper metric space. Let $\nu$ be a probability measure on $G$ of finite first moment, which means that

$$
\sum_{g \in G}|g| \nu(g)<\infty
$$

Define the entropy of $\nu$ by

$$
H(\nu):=-\sum_{g \in G} \nu(g) \log (\nu(g))
$$

Recall that we have

$$
H(\nu):=-\sum_{g \in G} \nu(g) \log (\nu(g)) \leq \log (2|S|) \sum_{g \in G}|g| \nu(g)+\log 2 .
$$

Indeed, let $a_{n}$ be the number of group elements of wordlength $n$. Then $a_{n} \leq|S|^{n}$. Define a probability measure $\nu^{\prime}$ on $G$ by $\nu^{\prime}(g)=1 /\left(2^{|g|+1} a_{|g|}\right)$. Then,

$$
H(\nu)-\sum_{g \in G} \nu(g) \log \left(2^{|g|+1} a_{|g|}\right)=-\sum_{g \in G} \nu(g) \log \frac{\nu(g)}{\nu^{\prime}(g)} \leq 0,
$$

where the inequality comes from Jensen's inequality (or $-\log t \leq 1 / t-1$ ) keeping in mind that both measures $\nu$ and $\nu^{\prime}$ are probability measures. The estimate (3) follows. By Kingman's subadditive ergodic theorem, the entropy of the random walk

$$
h(\nu):=\lim _{n \rightarrow \infty}-\frac{1}{n} \log \nu^{* n}\left(Z_{n}\right)=\inf _{n} \frac{1}{n} H\left(\nu^{* n}\right)
$$

exists and is constant almost surely. The vanishing of the entropy is related to bounded harmonic functions; it is proved in $[\mathrm{Av}],[\mathrm{D}]$ and $[\mathrm{KV}]$ that $h(\nu)=0$ if, 
and only if, the Poisson boundary is trivial. By applying (3) to $\nu^{* n}$, dividing by $n$, and letting $n \rightarrow \infty$, we get:

$$
h(\nu) \leq \log (2|S|) l(\nu) .
$$

Hence any measure $\nu$ with first moment on $G$ and with $l(\nu)=0$ has $h(\nu)=0$ and therefore only constant bounded $\nu$-harmonic functions. So if there are nonconstant bounded harmonic functions, $l(\nu)>0$. Otherwise, since $\nu$ is centered, $l(\nu)=0$ by Corollary 2 . This proves Corollary 3 .

Finally, taking as generators the set $S_{k}$ of elements with word length smaller than $k$, the new drift is not bigger than the old one divided by $k$. This shows that, for all $k, h(\nu) \leq \log \left(2\left|S_{k}\right|\right) l(\nu) / k$. Letting $k \rightarrow \infty$ yields $h \leq v l$, which is called the fundamental inequality in [Ve], and explains the role of subexponential growth in Corollary 5.

\section{REFERENCES}

[Al] G. Alexopoulos, On the mean distance of random walks on groups, Bull. Sci. Math. 111 (1987) 189-199

[Av] A. Avez, Entropie des groupes de type fini, C.R.Acad.Sci. Paris Sér.A-B 275 (1972) A1363-A1366

[C] T. K. Carne, A transmutation formula for Markov chains, Bull. Sci. Math. 109 (1985) 399-405

[D] Y. Derrienic, Quelques applications du théorème ergodique sous-additif, Astérisque, 74 (1980) 183-201

[E] A. Erschler, Boundary behaviour for groups of subexponential growth, Ann. Math. 160 (2004) 1183-1210

[G] Y. Guivarc'h, Sur la loi des grands nombres et le rayon spectral d'une marche aléatoire, Astérisque, 74 (1980) 47-98

[HP] U. Haagerup, A. Przybyszewska, Proper metrics on locally compact groups and proper affine isometric actions on Banach spaces, Preprint (2006)

[K] V. Kaimanovich, Poisson boundaries of random walks on discrete solvable groups, in Probability measures on groups, X (Oberwolfach, 1990), Plenum, New York, (1991) 205-238

[KV] V. Kaimanovich, A. Vershik, Random walks on discrete groups: boundary and entropy, Ann. Prob. 11 (1983) 457-490

[KM] A. Karlsson, G. Margulis, A multiplicative ergodic theorem and nonpositively curved spaces, Comm. Math. Phys., 208 (1999) 107-123

[KL1] A. Karlsson, F. Ledrappier, On laws of large numbers for random walks, Ann. Prob. 34 (2006) 1693-1706

[KL2] A. Karlsson, F. Ledrappier, The Liouville property and the speed of Brownian motion, in preparation (2007)

[M] P. Mathieu, Carne-Varopoulos bounds for centered random walks, Ann. Prob. 34 (2006) 987-1011

[Va] N. Th. Varopoulos, Long range estimates for Markov chains, Bull. Sci. Math. 109 (1985) 225-252

[Ve] A. Vershik, Dynamic theory of growth in groups: entropy, boundary, examples, Russian Math. Surveys 55 (2000) 667-733. 
Anders Karlsson

Department of Mathematics

Royal Institute of Technology

10044 Stockholm

Sweden

E-mail: akarl@math.kth.se

François Ledrappier

Department of Mathematics

University of Notre Dame

Notre Dame, IN 46556

U.S.A.

E-mail: fledrapp@nd.edu 